\title{
Efficient ion blocking in gaseous detectors and its application to gas-avalanche photomultipliers sensitive in the visible-light range
}

\author{
A. Lyashenko ${ }^{\mathrm{a}, *}$, A. Breskin ${ }^{\mathrm{a}}$, R. Chechik ${ }^{\mathrm{a}}$ J.M.F. dos Santos $^{\mathrm{b}}$, F.D. Amaro ${ }^{\mathrm{b}}$ and J.F.C.A. Veloso ${ }^{\mathrm{b}, \mathrm{c}}$ \\ ${ }^{a}$ Department of Particle Physics, The Weizmann Institute of Science, 76100 Rehovot, Israel \\ ${ }^{\mathrm{b}}$ Physics Department, University of Coimbra, 3004-516 Coimbra, Portugal \\ ${ }^{\mathrm{c}}$ Physics Department, University of Aveiro, Campus Universitário de Santiago, 3810-193 Aveiro, Portugal
}

\begin{abstract}
A novel concept for ion blocking in gas-avalanche detectors was developed, comprising cascaded micro-hole electron multipliers with patterned electrodes for ion defocusing. This leads to ion blocking at the $10^{-4}$ level, in DC mode, in operation conditions adequate for TPCs and for gaseous photomultipliers. The concept was validated in a cascaded visible-sensitive gas avalanche photomultiplier operating at atmospheric pressure of $\mathrm{Ar} / \mathrm{CH}_{4}(95 / 5)$ with a bi-alkali photocathode. While in previous works high gain, in excess of $10^{5}$, was reached only in a pulse-gated cascaded-GEM gaseous photomultiplier, the present device yielded, for the first time, similar gain in DC mode. We describe shortly the physical processes involved in the charge transport within gaseous photomultipliers and the ion blocking method. We present results of ion backflow fraction and of electron multiplication in cascaded patterned-electrode gaseous photomultiplier with $\mathrm{K}-\mathrm{Cs}-\mathrm{Sb}, \mathrm{Na}-\mathrm{K}-\mathrm{Sb}$ and $\mathrm{Cs}-\mathrm{Sb}$ visible-sensitive photocathodes, operated in DC
\end{abstract} mode.

Key words: gaseous photomultipliers, ion back-flow, ion feedback, bi-alkali photocathodes PACS: 29.40.Gx, 29.40.Ka, 29.40.Cs, 85.60.Gz, 85.60.Ha

\section{Introduction}

Controlling the back-flow of ions generated in gas avalanches has important consequences on the operation and properties of gaseous detectors. Avalanche ions induce space charge effects that limit the gain, counting-rate capability and localization properties of tracking detectors. The ion impact on photocathodes (PC) of gaseous photomultipliers (GPM) causes their permanent damage 112]; more seriously, it induces the emission of secondary electrons which, in turn, cause avalanche divergence, deterioration of timing and localization information and, most importantly, severe gain limits. All these consequences are included in the term Ion Feedback effects. GPMs sensitive in the UV range, with CsI PCs, do suffer some ion induced PC damage 2, but the ion-induced secondary electron emission probability is very low and does not limit their operation, even at high gain. There are numerous large-area CsI-GPMs, presently operating or under construction, in

\footnotetext{
* Corresponding author: tel. +972-8-934-2064, fax +972-8-934-2611, E-mail: alexey.lyashenko@weizmann.ac.il
}

many particle-physics experiments, e.g. COMPASS 3 and ALICE 4 at CERN and PHENIX 5] at BNL.

The ion feedback effects are particularly problematic in visible-sensitive GPMs, due to the high electron emission probability of bi-alkali and other PCs sensitive in the visible spectral range. The reader is referred to 6 for an extended discussion on this subject and references to recent works dealing with methods of ion back-flow reduction.

The only method for significantly reducing the ion backflow fraction (IBF) and reaching high-gain operation in visible-sensitive GPMs has been, so far, their operation in a gated mode 11. Our goal has been to find methods for efficiently reducing the IBF to permit the operation of visiblesensitive GPMs in DC mode with single-photon sensitivity. Obviously, large tracking TPCs will also benefit, as low IBF values would permit their stable DC operation.

In the present article, we report on our recent results on blocking of ion back-flow in cascaded micro-hole multipliers comprising GEMs and other patterned electrodes. We discuss the ion-induced secondary electron emission and provide solutions that permit, for the first time, the operation of visible-sensitive GPMs in DC mode, with gain of $10^{5}$. 


\section{Requirements for stable operation of visible-sensitive GPMs}

\subsection{General consideration on Ion back-flow and Ion feedback effects.}

While cascaded micro-hole multipliers, with their significant optical "opacity", efficiently block avalanche-photon feedback [7, they are less efficient in blocking the backflow of avalanche ions. The latter, originating from each avalanche stage in the cascaded multiplier, drift back to the GPMs' PC following the device field lines, and a major fraction of them follow the same paths (in opposite direction) of the initial photoelectrons and their successive avalanche electrons [6]. When impinging on the PC's surface they release secondary electrons; the latter initiate secondary avalanches, known as ion-feedback, which, by positive feedback mechanism diverge the proportional avalanche multiplication into discharge 8. An example of ion-feedback effect measured in a double-GEM multiplier with $\mathrm{K}-\mathrm{Cs}-\mathrm{Sb}$ visible-sensitive $\mathrm{PC}$ operating in $\mathrm{Ar} / \mathrm{CH}_{4}$ $(95 / 5)$ at 700 Torr (Fig. 1a), is the deviation of the gainvoltage curve from exponential (Fig. 1b). The measured gain, $\mathrm{G}_{\text {meas }}$, contains contributions from ion feedback and is described by:

$\mathrm{G}_{\text {meas }}=\frac{\mathrm{G}}{1-\gamma_{+} \cdot \mathrm{IBF} \cdot \varepsilon_{\text {extr }} \cdot \mathrm{G}}$

where $\gamma_{+}$is the ion induced secondary emission probability or the ion feedback probability, IBF is the ion back-flow fraction namely the fraction of ions, from all avalanche stages of the multiplier, flowing back to the $\mathrm{PC}$ (or to the drift region of a tracking detector), $\varepsilon_{\text {extr }}$ is the efficiency of extracting secondary electrons from the PC into the gas and G is the multiplier's gain without ion feedback. To avoid avalanche divergence into a spark, the above formula should fulfill $\gamma_{+} \cdot \mathrm{IBF} \cdot \varepsilon_{\text {extr }} \cdot \mathrm{G}<1$. Therefore, a gain of $10^{5}$, required for good single-photon sensitivity in GPMs, implies $\gamma_{+} \cdot \mathrm{IBF} \cdot \varepsilon_{\text {extr }}<10^{-5}$.

\subsection{Measurement of $\gamma_{+}$and $\varepsilon_{\text {extr }}$}

The extraction efficiency $\varepsilon_{\text {extr }}$ of secondary electrons is the fraction of electrons emitted from the PC and not scattered back (by collisions with gas molecules) into it 910. $\varepsilon_{\text {extr }}$ depends on the kinetic energy distribution of the electrons leaving the $\mathrm{PC}$, which has not yet been measured. The theoretical calculations of energy distribution and extraction efficiency $\varepsilon_{\text {extr }}$ of ion-induced secondary electrons from PCs are rather complex and differ from those of photoninduced ones 11. Such calculations are presently under way, in cooperation with T. Dias of Coimbra University, and will be the subject of a future publication.

In the absence of any knowledge of $\varepsilon_{\text {extr }}$, we have chosen to use $\gamma_{+}^{\text {eff }}=\gamma_{+} \cdot \varepsilon_{\text {extr }}$; the latter can be extracted from the experimental GPM's gain curve and its deviation
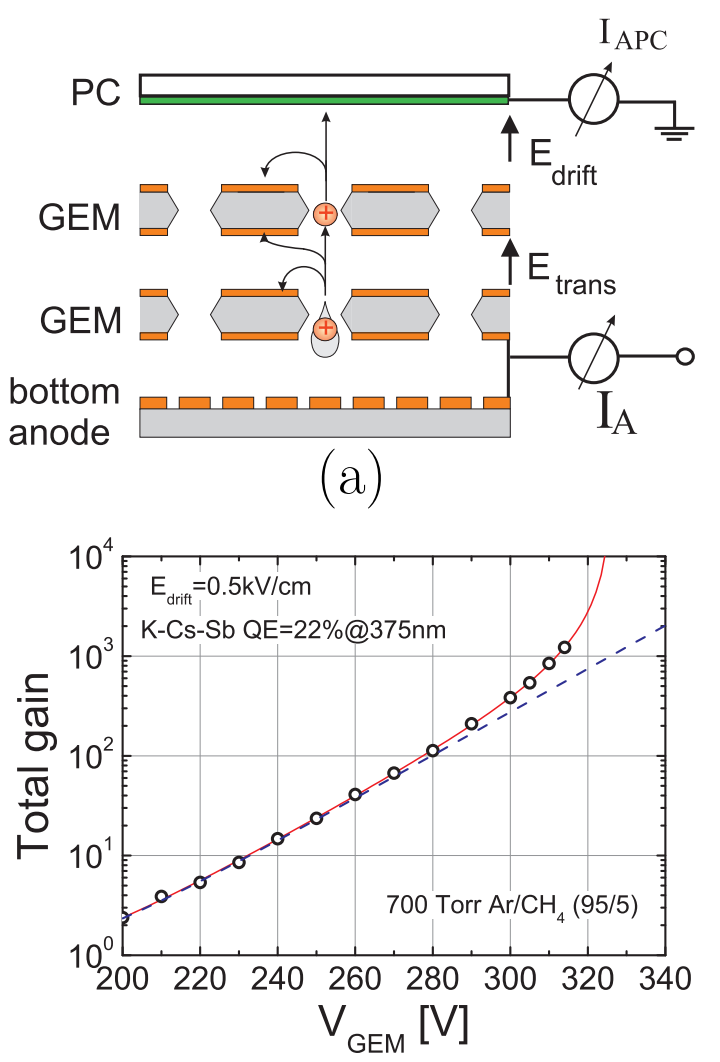

(b)

Fig. 1. (a) A double-GEM GPM coupled to a semitransparent photocathode; (b) gain-voltage characteristics measured in this GPM (see conditions in the figure, QE refers to vacuum) with CsI (dashed) and K-Cs-Sb (open circles) photocathodes. The divergence from exponential with $\mathrm{K}-\mathrm{Cs}-\mathrm{Sb}$ is due to ion feedback.

from exponential line. $\gamma_{+}^{\text {eff }}$ is defined as the effective ion induced secondary emission probability or the effective ion feedback probability. It was extracted from fitting the experimental gain curve $\mathrm{G}_{\text {meas }}$ by equation (1) (solid line in Fig. 1b. A significant deviation from the exponential gainvoltage characteristic (dashed line in Fig. 1b) is observed with the bi-alkali PC already at low gain. The IBF and G as a function of GEM voltage were measured in the same detector (geometry, gas and voltages), with a CsI PC, ensuring no ion feedback. The drift field between the $\mathrm{PC}$ and the top face of the first GEM was kept constant at $0.5 \mathrm{kV} / \mathrm{cm}$ throughout the entire measurements. In GPMs it provides about $60 \%$ extraction efficiency of photoelectrons in this gas $[8]$. The gain-voltage characteristics, like the one shown in Fig. 1b, were measured for K-Cs-Sb, Na-K-Sb and Cs-Sb PCs; they yielded $\gamma_{+}^{\text {eff }}$ values of $\sim 3 \cdot 10^{-2}$ for all these photocathodes. This study and the results will be discussed in more detail elsewhere.

\subsection{Requirements for $I B F$}

Establishing the effective ion feedback value $\gamma_{+}^{\text {eff }}$ with the visible sensitive PCs under investigation, we can set the 
limits on the IBF value needed for stable DC operation of visible-sensitive GPMs at a gain of $10^{5}$. Requiring

$\gamma_{+}^{e f f} \cdot \mathrm{IBF}<10^{-5}$, and using the estimated value

$\gamma_{+}^{e f f}=\sim 3 \cdot 10^{-2}$, the IBF value should be $<3.3 \cdot 10^{-4}$.

\section{IBF reduction in cascaded micro-hole multiplier structures}

A straight forward way to reduce the IBF is by lowering the drift field, since IBF decreases linearly with the drift field [12. However, in GPMs the drift field could not be too low because it controls, the photoelectron extraction into the gas, ; drift field values of the order of $0.5 \mathrm{kV} / \mathrm{cm} 8$ were generally applied in our GPMs filled with $\mathrm{Ar} / \mathrm{CH}_{4}(95 / 5)$.

A more detailed report on a comprehensive IBF reduction, study, carried out with a variety of cascaded microhole multipliers, can be found elsewhere [6]. All GPM detectors investigated had active areas of $30 \times 30 \mathrm{~mm}^{2}$; they were irradiated over a surface of $15 \mathrm{~mm}$ in diameter, at photon fluxes of about $10^{6} \frac{\mathrm{photons}}{\mathrm{sec} \cdot \mathrm{cm}^{2}}$. The core outcome of this study is that very low IBF values can be obtained with cascades combining GEMs and other patterned electrodes derived from the Micro-Hole \& Strip Plates (MHSP) [13. The latter comprised GEM-like holes and additional patterned strips on one of their faces, and they were operated in different modes regarding the voltages and orientation schemes $[6$ : MHSP, reversed-MHSP (R-MHSP) and flipped-reversedMHSP (F-R-MHSP). These schemes aim at reducing the IBF by diverting the ions and trapping them on the strips patterned on the surface. While the MHSP, placed at the end of the cascade, can divert and trap only part of the ions generated within its own avalanche stage, the other two types of electrodes can divert ions created in successive multiplying elements; therefore their incorporation in the cascade yielded better results. In a cascade comprising F-R-MHSP followed by GEM and MHSP (Fig. 2a), IBF values as low as $3 \cdot 10^{-4}$ were recorded [6]; this fulfills our requirement for stable DC operation at a gain of $10^{5}$ with visible-sensitive $\mathrm{PCs}$ (see Fig. 2b). We varied the inter-strip voltage on the bottom MHSP, to vary the total gain of the detector.

Following the success of the above study, and with our understanding of the operation mechanism of the MHSPlike electrodes [6, a new patterned micro-hole electrode named Cobra (Fig. 3) was developed with a geometry that is expected to improve the ion divergence away from the holes. It has thin anode electrodes surrounding the holes and creating strong electric field inside the holes (required for charge amplification); the more negatively biased cathode electrodes cover a large fraction of the area for better ion-collection as compared to the F-R-MHSP [6]. The concept of the Cobra electrode has been recently investigated. It was found that when introduced as a first element (with the patterned area pointing towards the photocathode), preceding two GEMs in the cascade (Fig. 4a), it drastically improved the ion trapping capability. The IBF as a
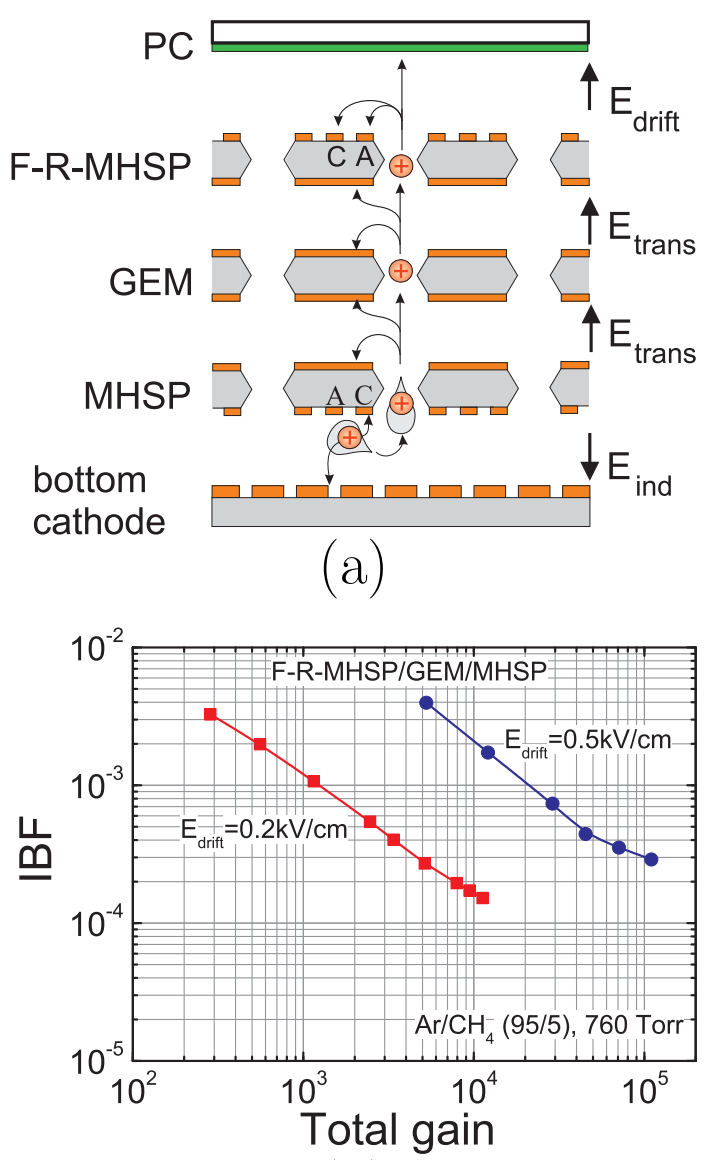

(b)

Fig. 2. (a) Scheme of the cascaded F-R-MHSP/GEM/MHSP multiplier coupled to a semitransparent photocathode; possible avalanche-ion paths are shown. (b) The IBF in correlation with the total gain of this GPM plotted for drift fields of $0.2 \mathrm{kV} / \mathrm{cm}$ (TPC conditions, squares) and $0.5 \mathrm{kV} / \mathrm{cm}$ (GPM conditions, circles).

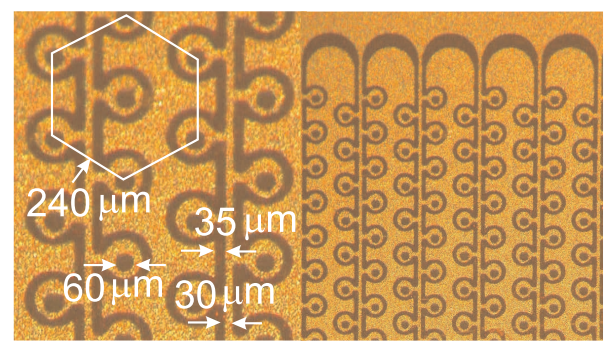

Fig. 3. A microscope photographs of one face of a "Cobra" micro-hole electrode with dimensions given in the figure. The other face is identical to a GEM.

function of voltage between electrodes on the top surface of Cobra is shown in Fig. 4b. In GPM conditions, with a drift field of $0.5 \mathrm{kV} / \mathrm{cm}$, we measured IBF values of $3 \cdot 10^{-6}$ which is 10,000 times lower than that of cascaded triple GEMs. In TPC conditions with a drift field of $0.2 \mathrm{kV} / \mathrm{cm}$ the same detector configuration provided IBF values as low as $2.7 \cdot 10^{-5}$. These are the lowest IBF values ever reached in gaseous detectors. However, while the F-R-MHSP yielded full photoelectron collection efficiency into the holes of the 

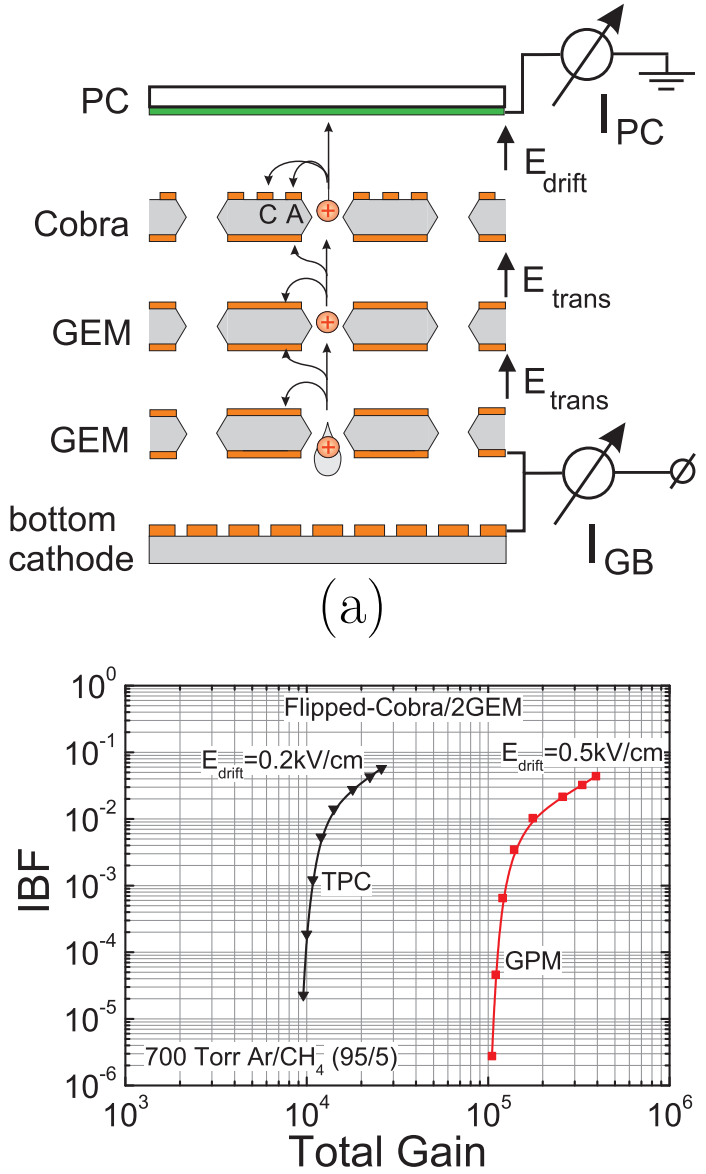

(b)

Fig. 4. (a) Scheme of cascaded Cobra/2GEM GPM with a semi-transparent photocathode; possible avalanche ions paths are also shown. (b) The IBF as a function of the total gain of the Cobra/2GEM cascaded detector for drift fields of $0.2 \mathrm{kV} / \mathrm{cm}$ (TPC conditions, triangles) and $0.5 \mathrm{kV} / \mathrm{cm}$ (GPM conditions, squares).

first cascade element, the Cobra, in its present geometry, had a limited electron collection efficiency of about $20 \%$. This can and should be improved by optimizing the geometrical parameters.

\section{DC operation of a visible-sensitive GPM with micro-hole multiplier cascades}

The operation of a visible-sensitive GPM in DC mode was investigated with a F-R-MHSP/GEM/MHSP cascaded multiplier, schematically shown in Fig. 2a. A photograph of the experimental detector is shown in Fig. 5 All the multiplier electrodes were mounted between ceramic spacers within a UHV vessel [8]. The photocathode was prepared and characterized in an adjacent vessel of the dedicated UHV system, and then transported with an UHV manipulator and placed in a stainless-steel holder above the detector. Details can be found in 14 and in 8 . The K-Cs-Sb PCs had typical quantum efficiency (QE) of $30 \%$ measured in vacuum at $375 \mathrm{~nm}$.
The details of the IBF measurements and results for this multiplier configuration were reported in 6 both in conditions for TPC and for GPM operation (Fig. 2b). Conditions for full efficiency of electron collection from the PC were confirmed and applied in all measurements

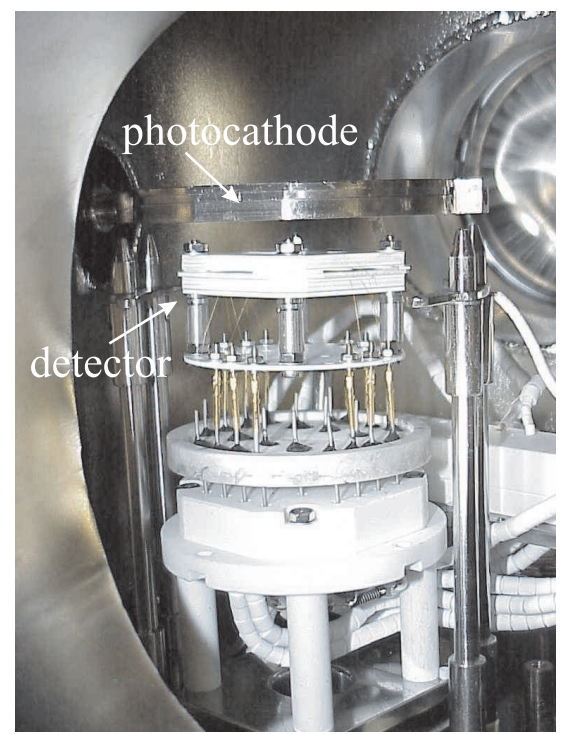

Fig. 5. Photograph of the F-R-MHSP/GEM/MHSP detector and the photocathode, mounted in the vacuum chamber.

In Fig. 6 we present gain-voltage characteristics for the cascaded GPM of Fig. 2a with a K-Cs-Sb PC and with a CsI $\mathrm{PC}$, for comparison. The measurements were carried out in $\mathrm{Ar} / \mathrm{CH}_{4}(95 / 5)$ at 700 Torr. The present semitransparent $\mathrm{K}$-Cs-Sb PC had a QE of $\sim 27 \%$ measured in vacuum at $375 \mathrm{~nm}$. Its QE value in the gas, with a drift-field of 0.5 $\mathrm{kV} / \mathrm{cm}$, was estimated to be $16 \%$ [8]. The solid and dashed curves in Fig. 6represent exponential fits to the data points measured with K-Cs-Sb and CsI PCs, correspondingly. In both cases the GPM could reach a gain of $10^{5}$ with no divergence from an exponential gain-voltage characteristic, indicating upon full suppression of ion feed-back effects.

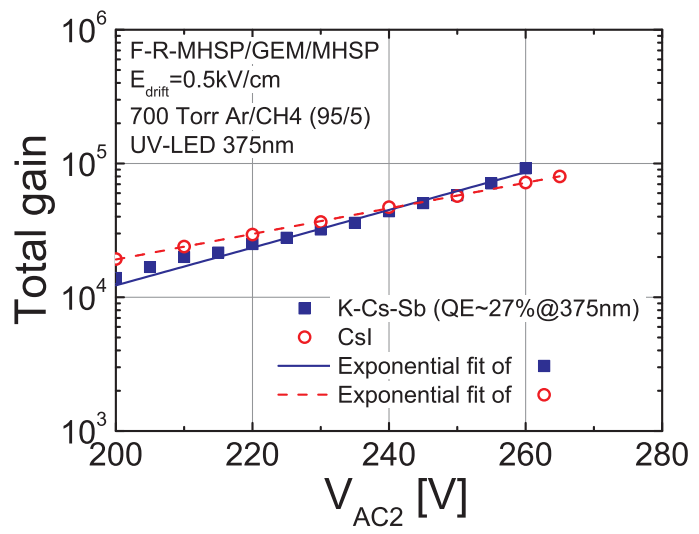

Fig. 6. Gain-voltage characteristics of the detector shown in Fig. 2a with a K-Cs-Sb (squares) and CsI (circles) photocathodes. The data was fitted with exponential functions; no divergence from exponential was observed. 700 Torr $\mathrm{Ar} / \mathrm{CH}_{4}(95 / 5) ; \mathbf{E}_{d r i f t}=0.5 \mathrm{kV} / \mathrm{cm}$. QE refers to vacuum. 


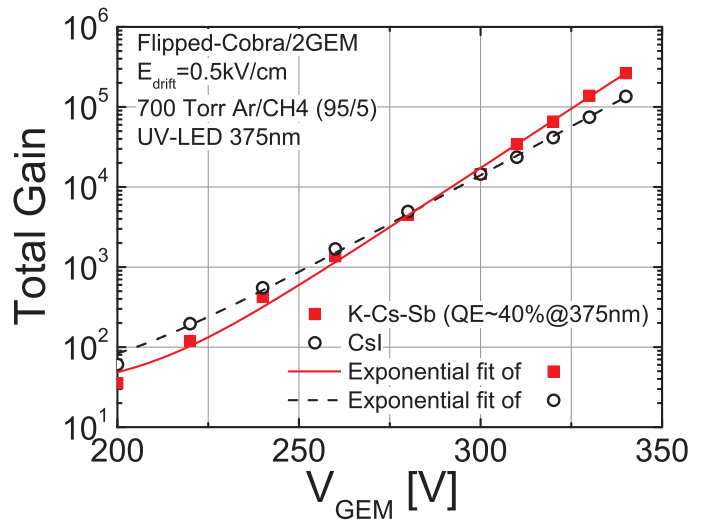

Fig. 7. Gain-voltage characteristics of the Cobra/2GEM cascaded GPM of Fig. 4a with K-Cs-Sb (squares) and CsI (circles) photocathodes. The data were fitted with exponential functions. 700 Torr $\mathrm{Ar} / \mathrm{CH}_{4}(95 / 5) ; \mathbf{E}_{\text {drift }}=0.5 \mathrm{kV} / \mathrm{cm}$. QE refers to vacuum.

The visible-sensitive GPM with a K-Cs-Sb PC coupled to the Cobra multiplier followed by two GEMs (Fig. 4a) was investigated in DC operation mode; the gain-voltage plots are shown in Fig. 7, in comparison with a CsI PC. The measurements were carried out in $\mathrm{Ar} / \mathrm{CH}_{4}(95 / 5)$ at 700 Torr. The semitransparent K-Cs-Sb PC had 40\% QE measured in vacuum at $375 \mathrm{~nm}$. The exponential fits to the data points measured with K-Cs-Sb and CsI PC are represented by solid and dashed curves, correspondingly. There were no feed-back effects as can be seen from the exponential shape of the gain-voltage curve.

\section{Conclusions}

Ion feedback in cascaded micro-hole gaseous detectors was studied with a variety of cascade elements and PCs, in conditions of TPC and of GPM operation. The effective ion feedback probability $\gamma_{+}^{\text {eff }}$ was measured in $\mathrm{Ar} / \mathrm{CH}_{4}(95 / 5)$ at 700 Torr and found to be $3 \cdot 10^{-2}$ for Na-K-Sb, K-Cs-Sb and $\mathrm{Cs}-\mathrm{Sb}$ photocathodes. Based on these measurements, the ion backflow fraction (IBF) required for stable DC operation of cascaded visible-sensitive gaseous photomultipliers (GPM) was estimated to be $3.3 \cdot 10^{-4}$.

Systematic ion blocking investigations with various patterned micro-hole cascaded multipliers yielded the required IBF values, at gain of $10^{5}$. The best results were recorded in a cascaded multiplier of a flipped reversed-bias microhole and strip plate flowed by a GEM and by a micro-hole and strip plate (F-R-MHSP/GEM/MHSP). This configuration yielded 100 fold lower IBF value than that measured in cascaded GEMs. This permits reaching stable operation conditions both for TPCs and for visible-sensitive GPMs operating in DC mode.

Even lower IBF values, of $3 \cdot 10^{-6}$ at a gain of $10^{5}$ and drift field $0.5 \mathrm{kV} / \mathrm{cm}$, was recorded in a cascade comprising a novel "Cobra" micro-hole patterned multiplier, followed by two GEMs. This IBF value is 10,000 times lower than that measured in cascaded GEMs. However, the electron collection efficiency of the present "Cobra" multiplier was only $20 \%$, which requires further optimization of its geometry.

A Visible-sensitive GPM with a F-R-MHSP/GEM/MHSP cascaded multiplier and a K-Cs-Sb photocathode, yielded, for the first time, stable operation at gains of $10^{5}$ in DC mode with full photoelectron collection efficiency and without any noticeable feedback effects. This is a breakthrough in the field of gaseous photomultipliers

Acknowledgments This work is partly supported by the Israel Science Foundation, grant No 402/05, by the MINERVA Foundation and by Project POCTI/FP/63962/2005 through FEDER and FCT (Lisbon). A. Breskin is the W.P. Reuther Professor of Research in The Peaceful Use of Atomic Energy.

\section{References}

[1] A. Breskin, et al., Ion-induced effects in GEM and GEM/MHSP gaseous photomultipliers for the UV and the visible spectral range, Nucl. Instr. and Meth. A 553 (2005) 46 and references therein.

[2] B. K. Singh, et al., CsBr and CsI UV photocathodes: new results on quantum efficiency and aging, Nucl. Instr. and Meth. A 454 (2000) 364.

[3] B. Ketzer, Micropattern gaseous detectors in the COMPASS tracker, Nucl. Instr. and Meth. A 494 (2002) 142.

[4] F. Piuz, et al., The CsI-based ring imaging detector for the ALICE experiment: technical description of a large prototype, Nucl. Instr. and Meth. A 433 (1999) 222.

[5] Z. Fraenkel, et al., A hadron blind detector for the PHENIX experiment at RHIC, Nucl. Instr. and Meth. A 546 (2005) 466.

[6] A. Lyashenko, et al., Further progress in ion back-flow reduction with patterned gaseous hole-multipliers, JINST 2 (2007) P08004 and references therein.

[7] D. Mörmann, et al., Operation principles and properties of the multi-GEM gaseous photomultiplier with reflective photocathode, Nucl. Instr. and Meth. A 530 (2004) 258.

[8] D. Mörmann, Study of novel gaseous photomultipliers for UV and visible light, $\mathrm{PhD}$ thesis, Weizmann Institute of Science, http://jinst.sissa.it/jinst/theses/2005_JINST_TH_004.jsp.

[9] A. Buzulutskov, et al., The GEM photomultiplier operated with noble gas mixtures, Nucl. Instr. and Meth. A 443 (2000) 164.

[10] J. Escada, et al., A Monte Carlo study of backscattering effects in the photoelectron emission from CsI into $\mathrm{CH}_{4}$ and $\mathrm{Ar}-\mathrm{CH}_{4}$ mixtures, JINST 2 (2007) P08001.

[11] H. Hagstrum, Theory of Auger neutralization of ions at the surface of a diamond-type semiconductor, Phys. Rev. 122 (1961) 83.

[12] A. Bondar, et al., Study of ion feedback in multi-GEM structures, Nucl. Instr. and Meth. A 496 (2003) 325.

[13] J. Veloso, et al., A proposed new microstructure for gas radiation detectors: The microhole and strip plate, Rev. Sci. Inst. A 71 (2000) 2371.

[14] M. Balcerzyk, et al., Methods of preparation and performance of sealed gas photomultipliers for visible light, IEEE Trans. Nucl. Sci. 50 (2003) 847. 